\title{
Surgical metastasectomy for renal cell carcinoma: which patients are the real candidates for surgery?
}

\author{
Satoshi Kato ${ }^{1}$, Satoru Demura ${ }^{1}$, Hideki Murakami ${ }^{2}$, Hiroyuki Tsuchiya ${ }^{1}$ \\ ${ }^{1}$ Department of Orthopaedic Surgery, Kanazawa University Graduate School of Medical Sciences, Kanazawa, Japan; ${ }^{2}$ Department of Orthopaedic \\ Surgery, Nagoya City University Graduate School of Medical Sciences, Nagoya, Japan \\ Correspondence to: Satoshi Kato, MD. Department of Orthopaedic Surgery, Kanazawa University Graduate School of Medical Sciences, 13-1 Takara- \\ machi, Kanazawa, 920-8641, Japan. Email: skato323@gmail.com. \\ Provenance: This is an invited article commissioned by the Section Editor Dr. Xiao Li (Department of Urology, Jiangsu Cancer Hospital, Jiangsu \\ Institute of Cancer Research, Nanjing Medical University Affiliated Cancer Hospital, Nanjing, China). \\ Comment on: Lyon TD, Thompson RH, Shah PH, et al. Complete Surgical Metastasectomy of Renal Cell Carcinoma in the Post-Cytokine Era. J \\ Urol 2019. [Epub ahead of print].
}

Submitted Oct 19, 2019. Accepted for publication Nov 28, 2019.

doi: 10.21037/atm.2019.11.139

View this article at: http://dx.doi.org/10.21037/atm.2019.11.139

Lyon et al. recently examined survival outcomes among patients with metastatic renal cell carcinoma (mRCC) treated with or without complete surgical metastasectomy (SM) during an era when targeted therapy and checkpoint inhibitors were available (1). They reported a greater 2 -year cancer-specific survival (CSS) in patients who underwent complete SM than in those who did not $(84 \%$ vs. $54 \%$, $\mathrm{P}<0.001)$ and that complete $\mathrm{SM}$ was associated with a significantly reduced likelihood of death from RCC after adjusting for age, sex, and the timing, number, and location of metastases. They concluded that metastasectomy may be considered for appropriately selected patients, even in the post-cytokine era.

Since the cytokine era, many studies on RCC have reported that SM has clinical benefits for various prognostic groups and is independently associated with prolonged survival (2-4). The current guidelines state that complete $\mathrm{SM}$ is recommended in appropriately selected patients $(5,6)$. However, in the era of targeted treatment, the outcomes of SM have not been well examined. Lyon et al. suggest that complete SM should continue to play a role in the management of mRCC patients despite availability of recently developed systemic therapies.

With the recent development of more effective systemic therapies, careful patient selection for SM is more important than ever. There is general consensus that several clinical and pathological factors, such as performance status $(4,7,8)$, disease-free interval $(2,4,7,8)$, abnormal laboratory data
$(2,7,8)$, sites of metastases (2,4), Fuhrman grade (2), and risk category in prognostic models $(3,7,8)$, affect prognosis and should be considered for the SM indication. A recent study reported that molecular subtypes might also be prognostic for outcomes after SM (9). SM is considered to have clinical benefits, including palliation or prevention of symptom and delay or withdrawal of systemic treatment, thereby avoiding deteriorated performance status and drug associated toxicities. Potential surgical stress and complication are also important to consider for the indication for SM. Complications and in-hospital mortality rates are not negligible in patients treated with targeted therapy who undergo surgical resection of mRCC $(10,11)$. After consideration of these issues, patients with a good indication for SM of mRCC should have the following features: (I) solitary or oligometastatic lesions, (II) symptomatic metastases deteriorating activities of daily living (ADL) and/or quality of life (QOL) or such an impending status, (III) resistance to radiotherapy and/or recently developed systemic therapies, and (IV) easy surgical accessibility and resectability with a lower rate of complications.

The incidences of mRCC in different anatomic sites vary. The metastatic site influences the symptoms, deterioration of general condition and ADL, and treatment strategy. More site-specific clinical factors that might have prognostic value for local treatment of metastases have been discussed in several systematic reviews (12-14). In this regard, there are some limitations to consider when interpreting the study by 
Lyon et al. In this study, more than the half of the patients undergoing SM had metastases in locations other than the lung, bone, non-regional lymph nodes, and liver, which are the four most common metastatic sites of RCC (15). They did not discuss the unusual mRCC distribution of the patients undergoing $\mathrm{CM}$ or the site-specific factors. Further, the study had the potential of a significant selection bias.

$\mathrm{SM}$ for mRCC is most commonly performed for pulmonary metastases, which are the most common metastases of RCC (15). Patients with metastases limited to the lung are the best responders to cytokine or targeted therapy (16). Resectable pulmonary lesions rarely cause deterioration of the general condition, ADL, or QOL. However, surgical accessibility with fewer major complications strengthens the benefits of pulmonary SM. Many studies have reported the clinical benefits of complete SM for pulmonary lesions and that a higher number of lesions $(4,17)$, concomitant mediastinal nodal metastases (17), and incomplete resection $(4,17)$ are associated with poor prognosis.

The bone is the second most common metastatic site of mRCC (15). Bone metastases tend to be highly destructive, resulting in pathologic fractures and spinal cord compression from lesions in the spine, which is the most affected bone site. These skeletal-related events severely compromise the performance status and QOL of the patients. A lowered performance status of patients with metastatic disease affects mortality directly as well as indirectly by hindering the delivery of systemic therapies. Generally, bone metastases are more resistant to radiotherapy and systemic therapy than other metastases (16). In terms of these factors, skeletal lesions have the best indication for SM if feasible. However, there are few comparative studies of SM of bone lesions $(18,19)$. Excisional surgery of bone metastases, especially in the spine, is an extraordinary and technically demanding surgery for general orthopedic and spine surgeons because the metastases are hypervascular and destructive, and reconstruction to support the operated lesion against the load is required after tumor resection in most cases. The surgeons in a well-experienced institution reported a case series of 36 consecutive patients undergoing complete spinal metastasectomy and its excellent clinical outcomes (20). However, for general surgeons, treatment goals for patients with bone metastases do not include complete SM. They are palliative surgeries combined nonsurgical treatments to preserve or restore of neurological function and improved pain control (21). Bone and brain metastases share the negative impact of complications after SM, and the introduction of stereotactic radiosurgery (SRS) and stereotactic body radiotherapy (SBRT) may improve treatment options in these patients. Although several comparative studies of SRS or SBRT for bone (21) and brain (22) metastases have been conducted, the interpretation of these studies is still controversial (12).

Despite the lymph nodes being the third most common metastatic site of RCC (15), isolated metachronous nodal metastases are rare, and most patients have other metastatic disease at multiple organs (23). There are few studies on such patients, and these studies report on only subgroups of patients who underwent SM for lymph node lesions compared with either no or incomplete resection. For liver and pancreatic metastases, the potential benefit needs to be balanced against the morbidity and mortality of surgical intervention for metastatic lesions. SM for these lesions should be carefully considered in patients with good performance status and completely resectable solitary metastases (24).

Lyon et al. also examined survival outcomes among patients with complete nonsurgical metastasis-directed treatment (MDT) for mRCC, which was defined in the study as radiation, radiofrequency ablation, and cryoablation. They compared survival in patients undergoing complete MDT and in those undergoing incompletes or no local therapy. The comparison of CSS and overall survival did not reach the threshold for statistical significance; however, the hazard ratio (HR) and confidence interval (CI) for CSS (HR 0.62; 95\% CI, $0.37-1.04, \mathrm{P}=0.07)$ suggest the potential for a significant relationship. There are also some limitations in this analysis to consider when interpreting the results. SRS and SBRT, which are representative of intensive nonsurgical MDT, were not included in the study. The definitions of complete and incomplete nonsurgical MDT and patient demographic data, including mRCC organ distribution, were not described. Conventional radiotherapy, SRS, and SBRT are applicable in primary RCC and mRCC in most organs. However, the feasibility and long-term oncologic control of percutaneous ablation, especially according to metastatic site and size, have not been well examined. A wide clinical application of effective nonsurgical MDT with fewer complications is anticipated, especially in patients in which complete SM is less feasible due to technical demand and a higher rate of major complications or in patients with multiple metastases. A retrospective study by Stenman et al. examined long-term overall survival after stereotactic 
radiotherapy or SM in $\mathrm{mRCC}$ patients in the era of targeted therapy. They reported that survival after stereotactic radiotherapy was comparable to that after $\mathrm{SM}$ and longer than expected, considering the high number of patients with adverse risk profiles (25).

Many questions regarding the clear benefits of CM remain unresolved and may change with advancements in cancer therapies. Future prospective studies, preferably with randomized designs and larger populations, are required to increase the quality of evidence regarding local treatment of mRCC, including not only SM but also non-surgical MDT. From a clinical perspective, the appropriate local treatment should be examined according to the metastatic organs, disease conditions, and possible genotype. Further, the possible benefits of survival and symptom control in patients with $\mathrm{mRCC}$ who are the real candidates for local treatment should be assessed. In clinical practice, patients with mRCC are treated with a multidisciplinary team approach involving the patient's medical oncologist, urologist, radiologist, and surgeons. Multimodal treatments combined with appropriate local treatment will surely prolong the survival of mRCC patients.

\section{Acknowledgments}

None.

\section{Footnote}

Conflicts of Interest: The authors have no conflicts of interest to declare.

Ethical Statement: The authors are accountable for all aspects of the work in ensuring that questions related to the accuracy or integrity of any part of the work are appropriately investigated and resolved.

\section{References}

1. Lyon TD, Thompson RH, Shah PH, et al. Complete Surgical Metastasectomy of Renal Cell Carcinoma in the Post-Cytokine Era. J Urol 2019. [Epub ahead of print].

2. Leibovich BC, Cheville JC, Lohse CM, et al. A scoring algorithm to predict survival for patients with metastatic clear cell renal cell carcinoma: a stratification tool for prospective clinical trials. J Urol 2005;174:1759-63; discussion 1763.

3. Eggener SE, Yossepowitch O, Kundu S, et al. Risk score and metastasectomy independently impact prognosis of patients with recurrent renal cell carcinoma. J Urol 2008;180:873-8.

4. Alt AL, Boorjian SA, Lohse CM, et al. Survival after complete surgical resection of multiple metastases from renal cell carcinoma. Cancer 2011;117:2873-82.

5. Motzer RJ, Jonasch E, Agarwal N, et al. Kidney Cancer, Version 2.2017, NCCN Clinical Practice Guidelines in Oncology. J Natl Compr Canc Netw 2017;15:804-34.

6. Ljungberg B, Bensalah K, Canfield S, et al. EAU guidelines on renal cell carcinoma: 2014 update. Eur Urol 2015;67:913-24.

7. Motzer RJ, Mazumdar M, Bacik J, et al. Survival and prognostic stratification of 670 patients with advanced renal cell carcinoma. J Clin Oncol 1999;17:2530-40.

8. Heng DY, Xie W, Regan MM, et al. Prognostic factors for overall survival in patients with metastatic renal cell carcinoma treated with vascular endothelial growth factortargeted agents: results from a large, multicenter study. J Clin Oncol 2009;27:5794-9.

9. Verbiest A, Couchy G, Job S, et al. Molecular Subtypes of Clear-cell Renal Cell Carcinoma are Prognostic for Outcome After Complete Metastasectomy. Eur Urol 2018;74:474-80.

10. Palumbo C, Pecoraro A, Knipper S, et al. Survival and complication rates of metastasectomy in patients with metastatic renal cell carcinoma treated exclusively with targeted therapy: a combined population-based analysis. Anticancer Res 2019;39:4357-61.

11. Meyer CP, Sun M, Karam JA, et al. Complications After Metastasectomy for Renal Cell Carcinoma-A Populationbased Assessment. Eur Urol 2017;72:171-4.

12. Dabestani S, Marconi L, Hofmann F, et al. Local treatments for metastases of renal cell carcinoma: a systematic review. Lancet Oncol 2014;15:e549-61.

13. Zaid HB, Parker WP, Safdar NS, et al. Outcomes following complete surgical metastasectomy for patients with metastatic renal cell carcinoma: a systematic review and meta-analysis. J Urol 2017;197:44-9.

14. Ouzaid I, Capitanio U, Staehler M, et al. Surgical metastasectomy in renal cell carcinoma: a systematic review. Eur Urol Oncol 2019;2:141-9.

15. Bianchi M, Sun M, Jeldres C, et al. Distribution of metastatic sites in renal cell carcinoma: a population-based analysis. Ann Oncol 2012;23:973-80.

16. Beuselinck B, Oudard S, Rixe O, et al. Negative impact of bone metastasis on outcome in clear-cell renal cell carcinoma treated with sunitinib. Ann Oncol 
2011;22:794-800.

17. Hofmann HS, Neef H, Krohe K, et al. Prognostic factors and survival after pulmonary resection of metastatic renal cell carcinoma. Eur Urol 2005;48:77-81; discussion 81-2.

18. Fuchs B, Trousdale RT, Rock MG. Solitary bony metastasis from renal cell carcinoma: significance of surgical treatment. Clin Orthop Relat Res 2005;431:187-92.

19. Du Y, Pahernik S, Hadaschik B, et al. Survival and prognostic factors of patients with renal cell cancer with bone metastasis in the era of targeted therapy: A singleinstitution analysis. Urol Oncol 2016;34:433.e1-8.

20. Kato S, Murakami H, Demura S, et al. Spinal metastasectomy of renal cell carcinoma: a 16-year single center experience with a minimum 3-year follow-up. J Surg Oncol 2016;113:587-92.

21. Hunter GK, Balagamwala EH, Koyfman SA, et al. The efficacy of external beam radiotherapy and stereotactic

Cite this article as: Kato S, Demura S, Murakami H, Tsuchiya H. Surgical metastasectomy for renal cell carcinoma: which patients are the real candidates for surgery? Ann Transl Med 2019;7(Suppl 8):S273. doi: 10.21037/atm.2019.11.139 body radiotherapy for painful spinal metastases from renal cell carcinoma. Pract Radiat Oncol 2012;2:e95-100.

22. Fokas E, Henzel M, Hamm K, et al. Radiotherapy for brain metastases from renal cell cancer: should whole-brain radiotherapy be added to stereotactic radiosurgery?: analysis of 88 patients. Strahlenther Onkol 2010;186:210-7.

23. Phillips CK, Taneja SS. The role of lymphadenectomy in the surgical management of renal cell carcinoma. Urol Oncol 2004;22:214-23.

24. Tanis PJ, van der Gaag NA, Busch OR, et al. Systematic review of pancreatic surgery for metastatic renal cell carcinoma. Br J Surg 2009;96:579-92.

25. Stenman M, Sinclair G, Paavola $P$, et al. Overall survival after stereotactic radiotherapy or surgical metastasectomy in oligometastatic renal cell carcinoma patients treated at two Swedish centres 2005-2014. Radiother Oncol 2018;127:501-6. 